\title{
An Adaptive Control For Wind Turbine
}

\author{
André Martinez, Fabien Lescher, Jing Yun Zhao \\ ERPA \\ EIGSI La Rochelle
}

26, rue de Vaux de Foletier - 17041 La Rochelle Cedex 1 - France

Phone/Fax number: 0033546458045 / 00335464580 10,

email: andre.martinez@eigsi.fr, fabien.lescher@eigsi.fr, jing-yun.zhao@eigsi.fr

\section{Brief introduction}

In this paper, an adaptive control is proposed to maximize the energy conversion for a wind turbine driven by a PWM (Pulse Width Modulation) Inverter. This paper focuses on the optimization of the current loops of the machine. Two different strategies of wind turbine management have been tested in steady and transient state (with the variation of the load). The principle of the optimization consists of minimizing Joule losses in PWM and Generator. The second strategy (adaptive) is implemented with successful results.

Key words: Adaptive Control, Variable Speed, Wind Turbine, PWM Inverter, current loops.

\section{Mathematical model of the system}

\section{A. Wind Energy: energy conversion}

Wind turbine using Permanent Magnet Synchronous Generator is represented figure 1:

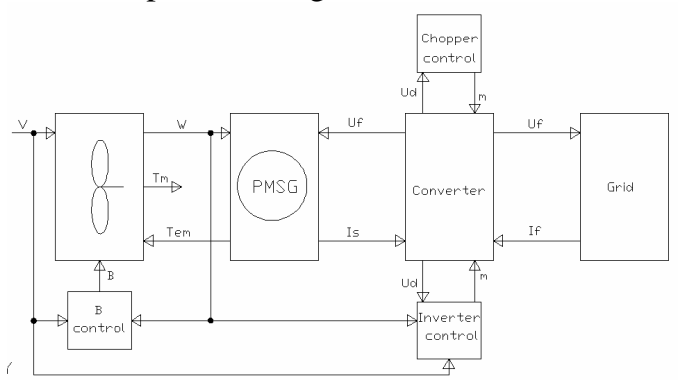

Fig. 1. Wind Turbine using PMSG

The structure most usually used consists in directly controlling the currents of phase by using 3 independent current loops [1, 2] , as shown figure 2 .

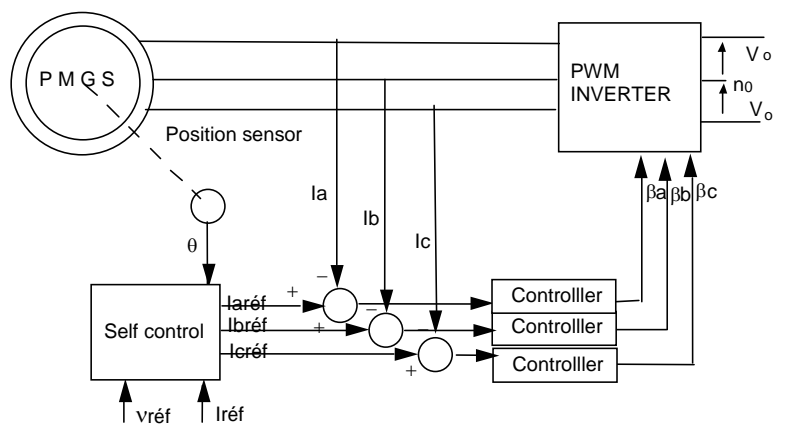

Fig. 2. Basic Voltage Source Converter structure

\section{Design of an adaptive control}

The efficiency of a wind turbine is dependent on the ratio of its blade tip speed, to the wind speed. Thus, as the speed of prevailing winds vary, so will the turbines efficiency. A method of extracting maximum energy from prevailing winds is therefore essential at all wind speeds. This can be achieved by controlling the turbines by means of an optimal control [3, 4].

For a Permanent Magnet Synchronous Generator the principle of the optimization consists of minimizing Joule losses in PWM and Generator. To do that, it is necessary to choose minimum phase current, considering fixed torque (figure 3).

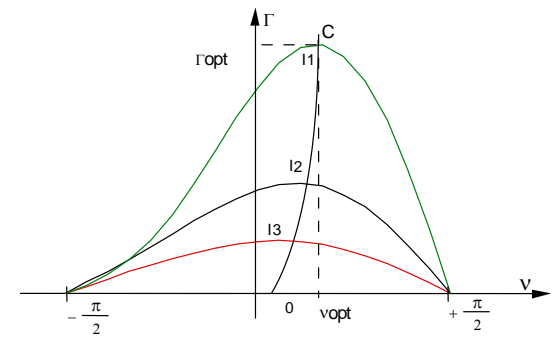

Fig. 3. $\Gamma(v)$ (with I1 > I2 > I3)

In the optimal control, the operating point is positioned on $\mathrm{C}$ curve (maximum torque and minimum current). The optimal algorithm will act on $v$ angle such as: $\left(\frac{\mathrm{dI}}{\mathrm{dv}}\right)_{\mathrm{C} \text { constant }}=0$

The principle of the optimal control is described figure 4 .

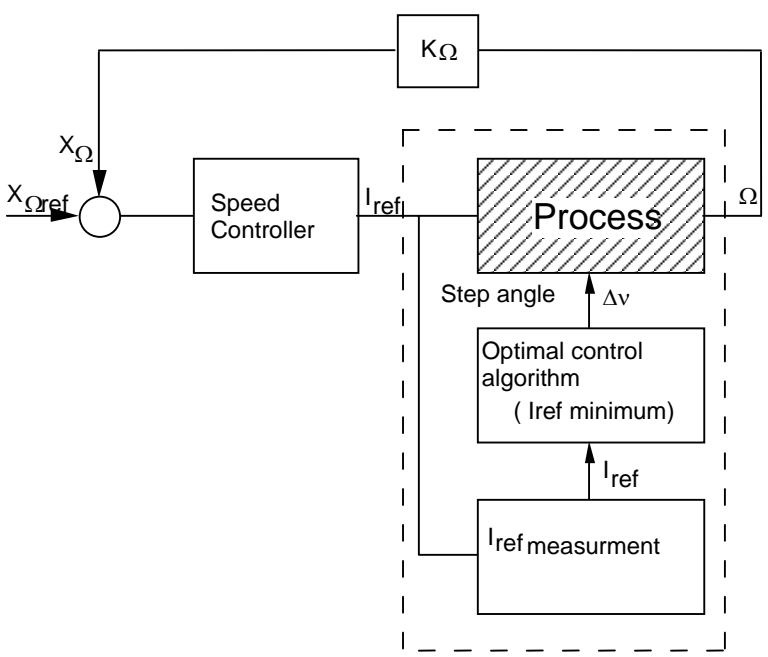

Fig. 4. Principle of the optimal control structure 


\section{Experimental results}

To test the performances of the last structure, we applied to the generator a load varying in crenels. Experimental results are obtained figure 5 :

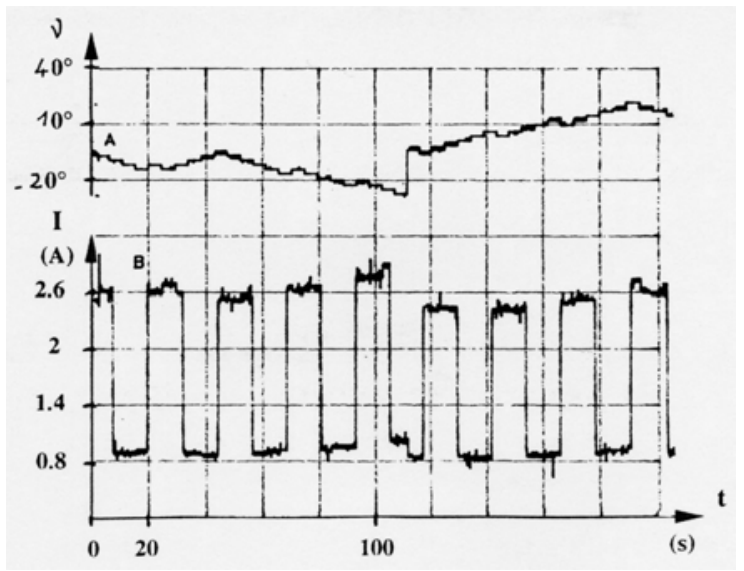

Fig. 5. I(t) and $v(\mathrm{t})$ (variable load)

The results obtained in this case, are really bad because the contribution of the various increments is no more prevalent in the current fluctuations and the algorithm diverges.

Notice: discontinuity in the $v$ angle on figure 11 corresponds to a reset of the $v$ angle by the control when its value exceeds $20^{\circ}$ (security).

Thus, to take into account the variations of current I, one solution consist in elaborating $\Delta v$ increment considering current/power of the generator.

\section{A. New structure of the optimal control}

A new structure of optimal control considering load variations has been implemented (figure 6).

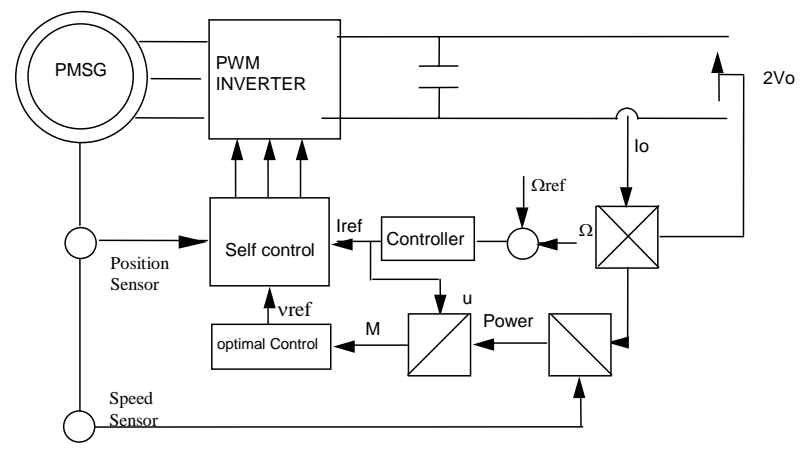

Fig. 6. New structure for the optimal control

The new algorithm is represented figure 6. If $\mid \mathrm{M}(\mathrm{k})$ Mref| is not higher than $\Delta$ Mo, that means that the $\Delta v$ step did not cause sufficiently significant change to make a decision on the new sign of the increment, then the old sign is kept until the sum of the steps is significant.

Figure 7 shows the results of this test. $v$ Angle oscillates around a stable average value.

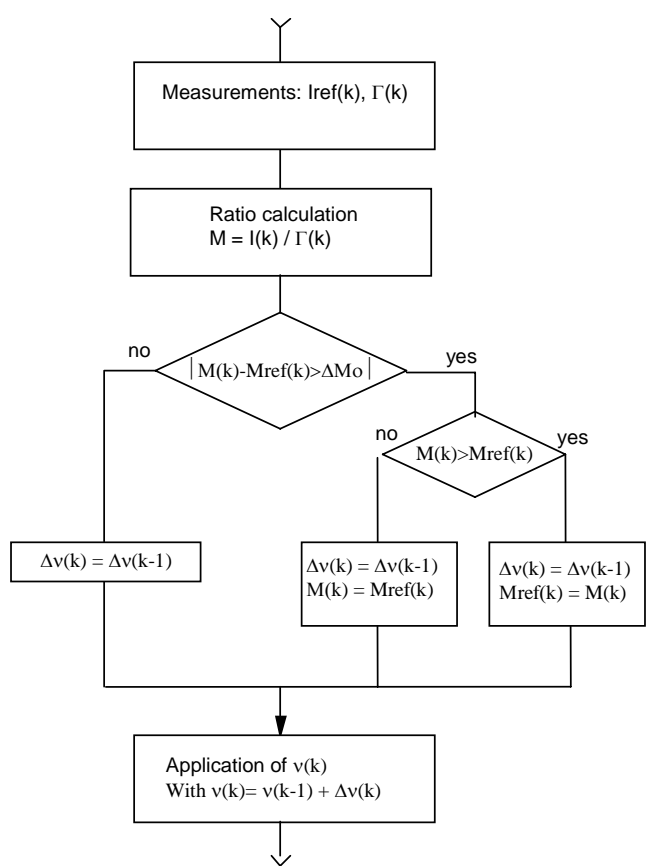

Fig. 6. Optimal control algorithm

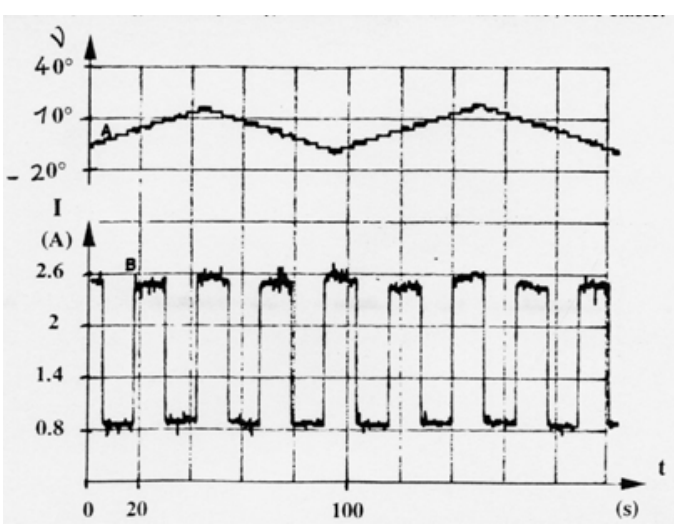

Fig. 7. $v$ and I (new MPPT algorithm)

\section{Conclusion}

An adapting optimal control is presented in this paper. A classical structure was implemented but results were not satisfactory, particularly in transient state (variation of the load), because of instability. This structure has been improved and advantages of the new control are verified when the load is varying.

\section{References}

[1] Slootweg, J. G., de Haan, S. W. H., Polinder, H., and Kling, W. L. 'Voltage Control Methods with Grid Connected Wind Turbines: a tutorial review'. Wind Engineering. Vol. 25, no. 6. 2001, pp. 353-365.

[2] L.H.Hansen, L.Helle, F.Blaajberg, E.RItchie, S.MunkNielsen, H.Bindner, P.Sorensen and B.Bak-Jensen, Conceptual survey of Generators and Power Electronics for wind turbines, Report, Riso National Laboratory, Roskilde, Denmark, Dec 2001.

[3] P.Novak, T.Ekelund,I.Jovik and B.Schmidtbauer. Modeling and control of variable speed wind turbine drive-system dynamics. IEEE Control Systems, 15(4):28-38, Aug 1995.

[4] W.Leonhard, Control of Electrical Drives, Springer-Verlag, 2001 